\title{
Near IR spectroscopy of the X-ray binary Circinus X-1
}

\author{
J. S. Clark ${ }^{1}$, P. A. Charles ${ }^{2}$, W. I. Clarkson ${ }^{2}$, and M. J. Coe ${ }^{2}$ \\ 1 Department of Physics and Astronomy, University College London, Gower Street, London, WC1E 6BT, UK \\ 2 Department of Physics and Astronomy, University of Southampton, Southampton SO17 1BJ, UK
}

Received 5 December 2002 / Accepted 6 January 2003

\begin{abstract}
We report on new $K$ band spectroscopy of the X-ray binary Cir X-1, which reveals the spectrum to be dominated by emission from $\mathrm{H}_{\mathrm{I}}, \mathrm{He}$ i and low-excitation metals. No features of photospheric origin are identifiable in the spectrum, preventing an unambiguous classification of the mass donor within the system. While the spectrum superficially resembles that of a highluminosity mid-B supergiant we consider that the magnitude and timescale of the photometric and spectroscopic variability renders such an identification highly unlikely. Instead we favour an explanation where the spectrum is dominated by emission from an accretion disc and/or accretion driven outflow(s), hence obscuring the spectrum of the underlying star.
\end{abstract}

Key words. stars: binaries: general - infrared: stars - stars: Individual: Cir X-1

\section{Introduction}

Since its discovery in the early 1970's (Margon et al. 1971) the nature of the X-ray binary Cir X-1 has remained elusive. An orbital period of $\sim 16.6$ days has been inferred from periodic modulation of the X-ray (Kaluzienski et al. 1976), near-IR (Glass 1994) and radio fluxes (Haynes et al. 1978) and the discovery of Type I X-ray bursts identified the compact object as a neutron star (Tennant et al. 1986). High optical reddening has made spectral classification of the counterpart impossible: the best optical spectra to date are dominated by asymmetric, emission lines of H $\mathrm{I}$ and He I (Johnston et al. 1999; Johnston et al. 2001; hereafter JFW99 \& J01 respectively), with no photospheric features evident.

The lack of spectral type has made determination of the mode of accretion occurring in Cir X-1 difficult. Murdin et al. (1980) interpreted the modulation of the X-ray and radio lightcurves as evidence for the direct accretion of material from the wind of a massive star $\left(M>20 M_{\odot}\right)$. Indeed the X-ray behaviour of Cir X-1 is similar to that of the 16.65-day High Mass X-ray Binary (HMXB) A0538-66, in which wind-fed accretion is enhanced by pseudo-Roche lobe overflow at periastron passage (Charles et al. 1983).

Conversely, JFW99 suggest that a similar scenario is also possible with an intermediate mass $\left(3-5 M_{\odot}\right)$ companion (IMXB). Furthermore, spectral-timing studies of Cir X-1 with RXTE/PCA (Shirey et al. 1999) show Z-source X-ray QPO behaviour, suggesting a Low Mass X-ray binary (LMXB) classification (cf. van der Klis 1995). However, the recent claim for a firm upper limit to the mass of the donor of $4.6 M_{\odot}$ by

Send offprint requests to: J. S. Clark, e-mail: jsc@star.ucl.ac.uk
Tauris et al. (1999) appears suspect in the light of an erroneous determination of the radial velocity of Cir X-1 (JFW99, J01; based on a doppler-shifted emission component). Similarly, the proposed association with the young supernova remnant G321.9-0.3, which would support a low-mass classification (e.g. Tauris et al. 1999) has recently been disproved by a proper motion study by Mignani et al. (2002). Clearly a consensus on the identity of Cir X-1 has yet to be reached. Here we present new $K$ band spectra of Cir X-1 taken in an attempt to provide a direct spectral classification of the mass donor.

\section{Observations and results}

Cir X-1 was observed once per night - for a total of $30 \mathrm{~min}$ on source exposure time - in service mode between 2002 July 26-28, during the commissioning of IRIS2, the new nearIR imager and spectrometer of the $3.92 \mathrm{~m}$ Anglo Australian Telescope. IRIS 2 uses a $1024 \times 1024$ Rockwell HAWAII-1 $\mathrm{HgCdTe}$ infrared detector, and used in the spectroscopic mode provides a resolution of $\sim 2400$. Observations of O8V (night 1) and A0V (nights 2 and 3) telluric standards at similar airmasses were also made. Unfortunately, photospheric $\operatorname{Br} \gamma$ is present in both spectra $(E W<2 \AA)$, complicating reduction. Removal of this feature from the telluric standards by division by a stellar spectrum of similar spectral type was found to be no better than a simple interpolation over the feature; we therefore caution against the reality of emission features in the wings of $\mathrm{Br} \gamma$ that may have been introduced by insufficient telluric correction.

The $K$ band spectrum (Fig. 1 and Table 1 ) of Cir X-1 is dominated by strong emission lines of $\mathrm{HI}$ and $\mathrm{He} \mathrm{I}$, with weak emission in the low-excitation Fe II $2.089 \mu \mathrm{m}$ line also present (Fig. 1). Problems with birefringence in the IRIS2 grisms 
(B. J. Boyle, private communication) led to blue-shifted components of the prominent emission features. To eliminate any possible confusion caused by this effect we have deconvolved the blue-shifted spectrum from the total and present the results in Fig. 1. Regrettably the strength of the blue-shifted $\mathrm{Br} \gamma$ component requires us to remove that section of the spectrum around $2.15 \mu \mathrm{m}$ to avoid any ambiguity in spectral features. We do, however, note the presence of a possible emission line at $2.138 \mu \mathrm{m}$ corresponding to $\mathrm{Mg}$ II at the edge of this gap in our data from July 28. Weak He II (10-7) $2.189 \mu$ m emission is also seen in the July 26 spectrum, although no emission is observed in other high excitation metallic transitions such as C IV or $\mathrm{N}$ III. We also find no evidence for photospheric features from low excitation metals or molecular bands. While the $\mathrm{Mg}_{\mathrm{I}}$ and $\mathrm{Al}$ I transitions around $2.11 \mu \mathrm{m}$ will clearly be hidden by emission in the He $\mathrm{I} 2.112 / 3 \mu \mathrm{m}$ lines, the 2.189 and $2.209 \mu \mathrm{m}$ $\mathrm{Si}$ II lines are not present, which we would expect to be in absorption if the temperature was $<10000 \mathrm{~K}$. Likewise, we can find no evidence for the Na I doublet $(2.20624 / 2.20897 \mu \mathrm{m})$ the Ca I triplet $(2.26141 / 2.26311 / 2.26573 \mu \mathrm{m})$ or the ${ }^{12} \mathrm{CO}$ and ${ }^{13} \mathrm{CO}$ molecular bandheads longwards of $2.29 \mu \mathrm{m}$, which are characteristic of late $(\mathrm{G}-\mathrm{M})$ spectral types.

Given the significant flaring in the X-ray-radio continuum, the observations were timed to coincide with the periastron passage of the neutron star (Fig. 2 and Table 1). No evidence for shifts in lines accompanying the periastron passage was found; indeed the spectral morphology - both in terms of lines present and line flux ratios - is remarkably similar in the three observations. The only night to night variations are the disappearance of He II $2.189 \mu \mathrm{m}$ emission on nights 2 and 3 and an increase in the strength of the remaining emission features by $\sim 30$ per cent between nights 1 and 2 . Unfortunately only one previous observation of Cir X-1 has been made (JFW99; Table 1 and Fig. 3); however it is clear that the strength of the He I $2.058 \mu \mathrm{m}$ and $\operatorname{Br} \gamma$ lines has decreased dramatically between the 2 epochs of observations, mirroring the long-term decrease in the $\mathrm{H} \alpha$ flux that has accompanied the reduction in X-ray luminosity (Johnston et al. 2001).

\section{Discussion}

Given the rich emission line spectrum of Cir X-1 a natural assumption would be that it arises from a dense stellar wind from a massive star. Comparison to the spectra of $\mathrm{O}$ stars from Hanson et al. (1996) shows that the lack of C IV and N III emission lines precludes a classification earlier than $\sim 06$, while emission in $\mathrm{Br} \gamma$ and $\mathrm{He}$ II excludes later $\mathrm{O}$ stars. Furthermore, the presence of strong $\mathrm{Br} \gamma$ emission, weak He II and narrow emission lines precludes a classification as a chemically evolved Wolf Rayet (e.g. Figer et al. 1997).

The remaining possibilities for a massive companion of recognised classification are classical Be star or B supergiant. In Fig. 3 we plot the spectrum of the classical Be X-ray binary XTE 1946+274 (Verrecchia et al. 2002) for comparison; note the superficial similarities to our spectra of Cir X-1. However we discount this as a satisfactory classification on the basis of the strength of the emission features in the spectrum presented by JFW99; $\mathrm{Br} \gamma$ and He I $2.058 \mu \mathrm{m}$ emission in both isolated

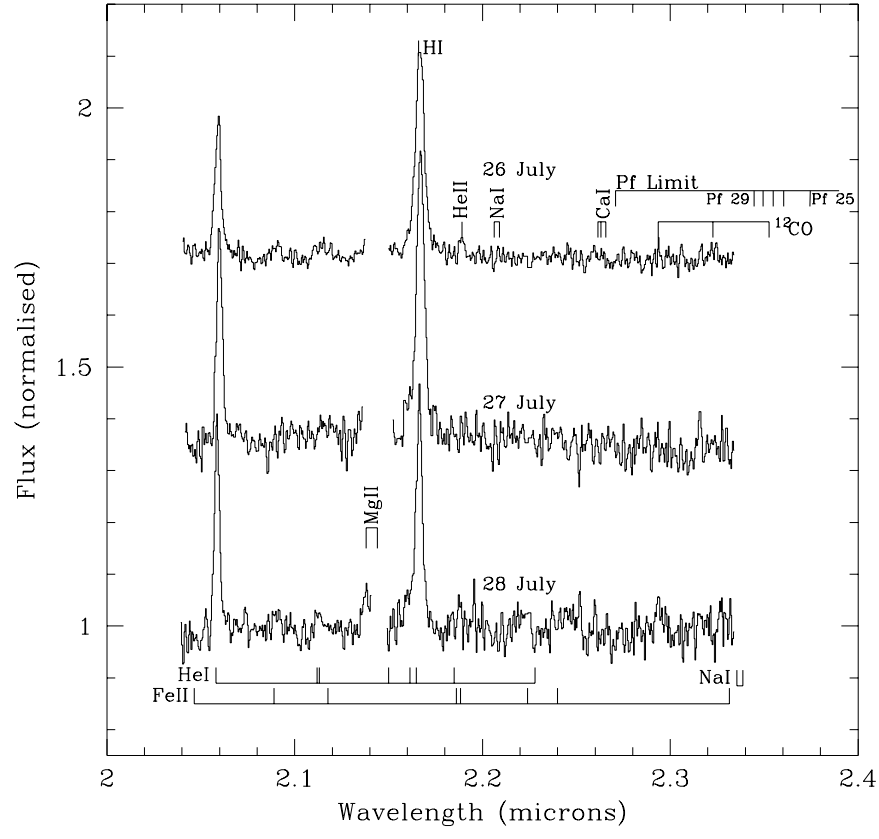

Fig. 1. $K$ band spectra of Cir X-1, with the rest wavelengths of important transitions within the spectral window indicated.

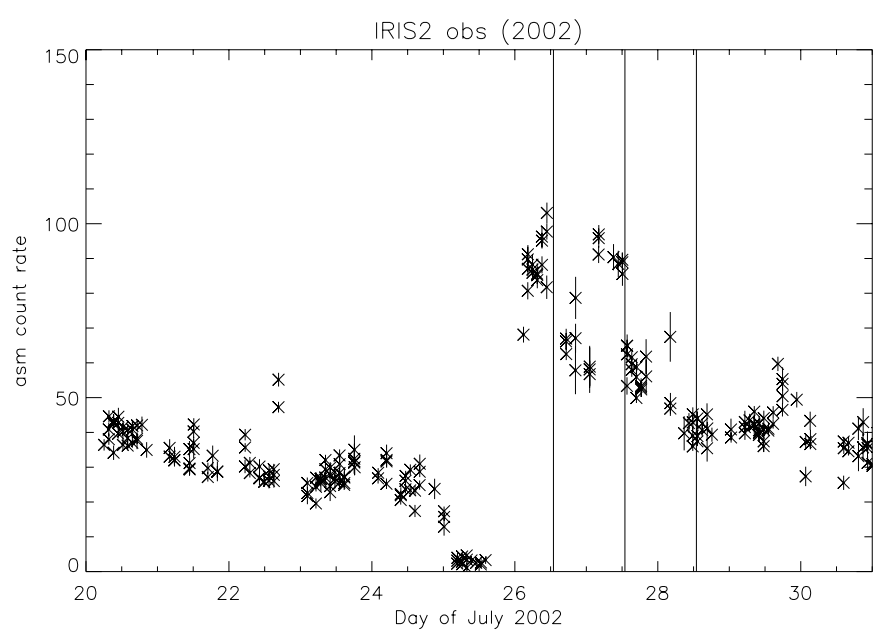

Fig. 2. XTE ASM X-ray lightcurve of Cir X 1 with the position of the IR observations indicated by vertical lines.

Be stars (Clark \& Steele 2000) and Be/X-ray binaries (Clark et al. 1999; Hanson et al. 1996) is uniformly less than 20 per cent of the strength observed in Cir X-1 at this time (the same is true of the strength of the optical $\mathrm{H} \alpha$ line of Cir X-1 prior to 2000; JFW99).

The final option for a high mass counterpart then appears to be a luminous B supergiant, with a high mass loss rate to drive the observed lines strongly into emission. In Fig. 3 we overplot the spectrum of Cir X-1 with the synthetic spectrum of a mid-B supergiant constructed with the non-LTE code of Hillier \& Miller (1998) for comparison. Since the available data do not let us constrain the wind velocity law (we would require an observation of a strong resonance line such as He I $1.083 \mu \mathrm{m}$ ) we have not attempted to formally fit the spectrum; rather we present a representative spectrum with parameters appropriate 
Table 1. Suggested identifications for emission features in Cir X-1 (wavelengths given in $\mu \mathrm{m}, E W \mathrm{~s}$ in $-\AA$ ). We estimate errors in the $E W$ to be of the order of $\pm 10 \%$ of the quoted value for the strong ( $E W \geq 10 \AA$ ) lines and $\pm 25 \%$ for transitions weaker than this. em denotes those lines that are in emission but are too weak to measure an $E W$ for. Note that in the spectrum of Johnston et al. (1999) obtained on 1997 June 11 $(\phi \sim 0.93)$ only $\mathrm{He}_{\mathrm{I}} 2.058 \mu \mathrm{m}(E W=107 \pm 9 \AA)$ and $\mathrm{Br} \gamma(E W=100 \pm 9 \AA)$ were identified as being strongly in emission, although the presence of a weaker broad emission feature at $\sim 2.1 \mu \mathrm{m}$ is suggested.

\begin{tabular}{llccc}
\hline \hline $\begin{array}{l}\text { Central } \\
\text { Wavelength }\end{array}$ & $\begin{array}{l}\text { Suggested } \\
\text { Transition }\end{array}$ & $\mathrm{N} 1(\phi=0.07)$ & $\begin{array}{c}E W(\AA) \\
\mathrm{N} 2(\phi=0.13)\end{array}$ & $\mathrm{N} 3(\phi=0.17)$ \\
\hline 2.0597 & $\mathrm{He}$ I $\left(2 \mathrm{~s}^{1} \mathrm{~S}-2 \mathrm{p}^{1} \mathrm{P}^{0}\right) 2.058$ & 11.8 & 15.3 & 15.2 \\
2.0907 & $\mathrm{Fe}$ II $\left(\mathrm{z}^{4} \mathrm{~F}_{3 / 2}-\mathrm{c}^{4} \mathrm{~F}_{3 / 2}\right) 2.089$ & 1.8 & - & 1.9 \\
2.113 & $\mathrm{He}_{\mathrm{I}}\left(3 \mathrm{p}^{3} \mathrm{P}^{0}-4 \mathrm{~s}^{3} \mathrm{~S}\right) 2.112+$ & $\mathrm{em}$ & $\mathrm{em}$ & 2 \\
& $\mathrm{He}$ I $\left(3 \mathrm{p}^{1} \mathrm{P}^{0}-4 \mathrm{~s}^{1} \mathrm{~S}\right) 2.113$ & $\mathrm{bl}$ & $\mathrm{bl}$ & $\mathrm{bl}$ \\
2.1665 & $\mathrm{Br} \gamma 2.1661$ & 24.2 & 32.2 & 19.5 \\
2.1887 & $\mathrm{He}$ II $(10-7) 2.189$ & 1.3 & - & - \\
\hline
\end{tabular}

for such an object $\left(L=5 \times 10^{5} L_{\odot}, \dot{M}=10^{-5} M_{\odot} \mathrm{yr}^{-1}\right.$, $v_{\infty} \sim 150 \mathrm{~km} \mathrm{~s}^{-1}, T_{\text {eff }}=15 \mathrm{kK}$; Clark et al. in prep.). Given that we have made no attempt to optimise the fit to the observational data, the synthetic spectrum shows a surprising similarity (Fig. 3) - the major exception being our inability to drive the He II $2.189 \mu \mathrm{m}$ line into emission.

Despite the similarity between observed and synthetic spectra we are uncomfortable suggesting such a classification, given the nature and magnitude of the recurrent photometric and spectroscopic variability of Cir X-1. In particular, while a subset of massive stars in this region of the HR diagram are known to be highly variable (the "Luminous Blue Variables"; LBVs), an exceptionally high mass loss rate would be required to explain the 1997 spectrum $\left(\gg 10^{-4} M_{\odot} / \mathrm{yr}\right)$; such stars are both highly luminous $\left(\geq 10^{6} L_{\odot}\right)$ and exceptionally rare (currently only 3 are known in the galaxy, Clark et al. in prep.). Moreover, while LBVs are known to be highly variable, changes of 23 mag (so called "Giant Eruptions") occur over timescales of years - decades (e.g. Humphreys \& Davidson 1994); very different from the rapid, periodic near-IR flaring observed in Cir X-1 by Glass (1994).

While it appears unlikely that the mass donor in Cir X-1 is an $\mathrm{OB}$ star, there are no spectroscopic features present that would unambiguously allow us to identify the donor as a lowmass star, such as the Na I doublet, $\mathrm{Ca}$ I triplet and $\mathrm{CO}$ bandheads that are seen in absorption for both isolated late (G-M) stars (Kleinmann \& Hall 1986; Wallace \& Hinkle 1997) and LMXB (e.g. Bandyopadhyay et al. 1999). This would suggest that if we are observing the uncontaminated stellar spectrum, the mass donor is of spectral type $\mathrm{F}$ or earlier, implying an IMXB classification.

However given the IR-flaring at periastron we suspect that the spectrum is subject to significant contamination that could serve to mask any photospheric features that might be present; indeed observations of the LMXB GRS 1915+105 over a number of years failed to identify the weak photospheric features of the K-M mass donor (Greiner et al. 2001). Possible sources of continuum emission which could veil the spectrum of the companion include contributions from an accretion disc (cf. Sco X-2; Bandyopadhyay et al. 1999), synchrotron emission from the putative jet (e.g. Fender et al. 1998) or thermal bremsstrahlung from an accretion driven outflow (cf. SS 433;

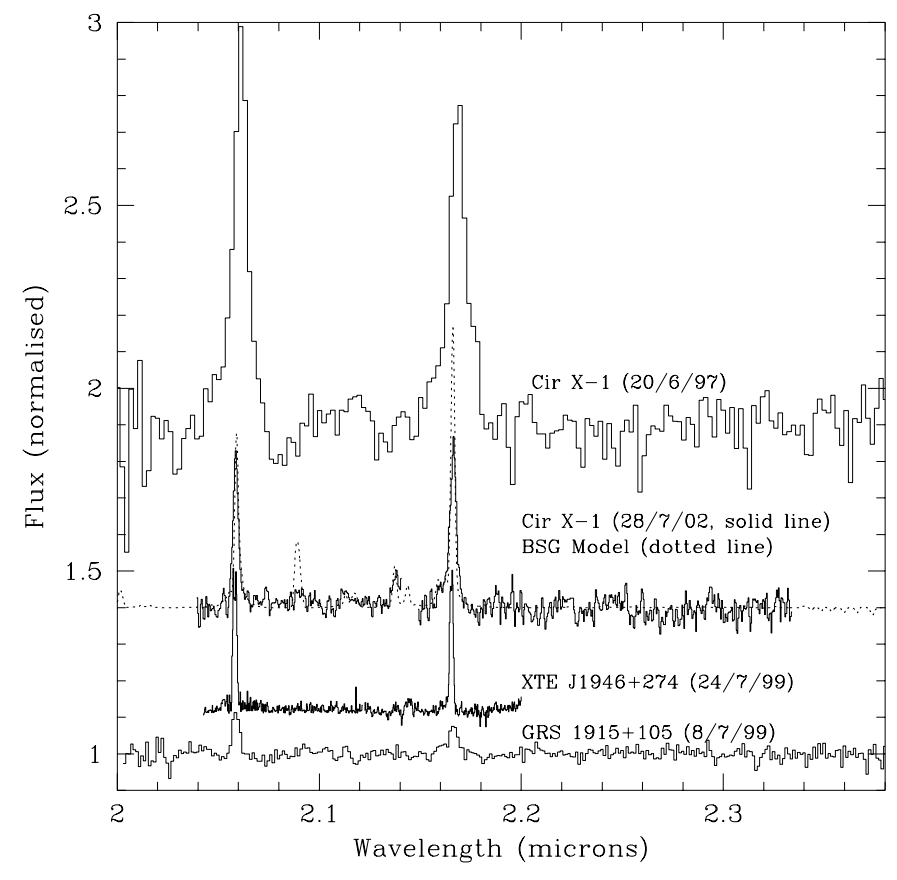

Fig. 3. Mean $K$ band spectrum of Cir X-1 compared to a synthetic spectrum of a mid-B supergiant (dotted line). The spectra of the Be/X-ray binary XTE 1946+274 (Verrecchia et al. 2002), the low mass X-ray binary GRS 1915+105 (Harlaftis et al. 2001) and the earlier spectrum of JFW99 are also shown for comparison - note the dramatic change in line strengths of Cir X-1 between the 2 epochs.

Gies et al. 2002). In such a scenario, an origin in an accretion disc and/or accretion driven outflow is also suggested for the strong emission lines. J01 report double peaked $\mathrm{H} \alpha$ profiles which they associate with doppler splitting in a rotating accretion disc, while a fast (200-1800 $\mathrm{km} \mathrm{s}^{-1}$ ) accretion-driven wind is known to be present in the system from observations of X-ray P Cygni profiles (e.g. Brandt \& Schulz 2000).

\section{Conclusions}

The $\mathrm{K}$ band spectrum of Cir X-1 is found to be dominated by emission from $\mathrm{HI}, \mathrm{He}_{\mathrm{I}}$ and low excitation metals. No photospheric features from low excitation atomic species or molecules are identifiable. While the morphology of the 
spectrum remains constant during the period of the observations - which coincide with the assumed periastron passage of the neutron star - variation in the strength of the emission lines was observed. Comparison to the single $K$ band spectrum of JFW99 similarly reveals long term variability in line strength that mirrors the variability observed in the optical spectrum and is apparently correlated with the long-term decrease in X-ray luminosity.

The lack of photospheric features prevents an unambiguous determination of the spectral type and luminosity class of the mass donor. While our present IR spectrum is consistent with a classification of the mass donor as a mid-B supergiant with high luminosity and mass loss rate, the periodic continuum flaring and previous strength of the $\mathrm{H}_{\mathrm{I}}$ and $\mathrm{He}_{\mathrm{I}}$ emission lines are inconsistent with a purely stellar origin. This implies that irrespective of the mass of the donor the emission components of the spectrum are at least partially driven by energy liberated by accretion.

The absence of low-excitation atomic or molecular photospheric features suggests a spectral type of $\mathrm{F}$ or earlier for the mass donor (and hence an IMXB classification) if no veiling of the spectrum due to a non-stellar source of continuum radiation is present. However, given the IR flaring at periastron observed by Glass (1994) we believe it is likely that excess IR emission - which could obscure the photospheric features from a low-intermediate mass companion - is present in our spectra. Several non-stellar sources of near IR-continuum emission potentially exist within Cir X-1, including an accretion disc and/or disc wind and the outflow responsible for the radio emission. Given the high X-ray luminosity and accretion rate during periastron, an origin for both the line and (transient) continuum emission in a mass outflow driven by accretion appears attractive. Such an interpretation is supported by the detection of P Cygni emission line profiles in the X-ray spectrum, which - given the low wind velocity associated with a putative blue supergiant companion - appear to be unambiguously associated with a high velocity accretion-driven outflow irrespective of the mass of the stellar companion.

Indeed a rich emission-line spectrum originating in an accretion driven outflow of matter appears to be an inescapable consequence of accretion at $\sim$ Eddington rates - cf. CI Cam (Clark et al. 2000), A0538-66 (Charles et al. 1983) and SS 433 (Gies et al. 2002), in which the spectra during outburst are dominated by emission from an accretion-powered wind, despite the early spectral type $(\mathrm{O}, \mathrm{B}$ or $\mathrm{A})$ and large $\left(>10^{4} L_{\odot}\right)$ luminosity proposed for the mass donors.

\section{References}

Bandyopadhyay, R. M., Shahbaz, T., Charles, P. A., \& Naylor, T. 1999, MNRAS, 306, 417

Brandt, W. N., \& Schulz, N. S. 2000, ApJ, 544, L123

Charles, P. A., Booth, L., Densham, R. H., et al. 1983, MNRAS, 202, 657

Clark, J. S., Steele, I. A., Fender, R. P., \& Coe, M. J. 1999, A\&A, 348, 888

Clark, J. S., \& Steele, I. A. 2000, A\&AS, 141, 65

Clark, J. S., Miroshnichenko, A. S., Larionov, V. M., et al. 2000, A\&A, 356,50

Fender, R. P., Spencer, R., Tzioumis, T., et al. 1998, ApJ, 506, L121

Figer, D. F., McLean, I. S., \& Najarro, F. 1997, ApJ, 486, 420

Gies, D. R., McSwain, M. V., Riddle, R. L., et al. 2002, ApJ, 566, 1069

Glass, I. S. 1994, MNRAS, 268, 742

Greiner, J., Cuby, J. G., McCaughrean, M. J., Castro-Tirado, A. J., \& Mennickent, R. E. 2001, A\&A, 373, L37

Harlaftis, E. T., Dhillon, V. S., \& Castro-Tirado, A. 2001, A\&A, 210

Hanson, M. M., Conti, P. S., \& Rieke, M. J. 1996, ApJS, 107, 281

Haynes, R. F., Jauncey, D. L., Murdin, P. G., et al. 1978, MNRAS, 185,661

Hillier, D. J., \& Miller, D. L. 1998, ApJ, 496, 407

Humphreys, R. M., \& Davidson, K. 1994, PASP, 106, 1025

Johnston, H. M., Fender, R., \& Wu, K. 1999, MNRAS, 308, 415 (JFW99)

Johnston, H. M., Wu, K., Fender, R., \& Cullen, J. G. 2001, MNRAS, 328, $1193(\mathrm{~J} 01)$

Kaluzienski, L. J., Holt, S. S., Boldt, E. A., \& Serlemitsos, P. J. 1976, ApJ, 208, L71

Kleinmann, S. G., \& Hall, D. N. B. 1986, ApJS, 62, 501

Margon, B., Lampton, M., Bowyer, S., \& Cruddace, R. 1971, ApJ, 169, L23

Mignani, R. P., De Luca, A., Caraveo, P. A., \& Mirabel, I. F. 2002, A\&A, 386, 487

Murdin, P., Jauncey, D. L., Haynes, R. F., et al. 1980, A\&A, 87, 292

Shirey, R. E., Bradt, H. V., \& Levine, A. M. 1999, ApJ, 517, 472

Tauris, T. M., Fender, R. P., van den Heuvel, E. P. J., Johnston, H. M., \& Wu, K. 1999, MNRAS, 310, 1165

Tennant, A. F., Fabian, A. C., \& Shafer, R. A. 1986, MNRAS, 221, 27

van der Klis, M. 1995, X-ray binaries, ed. W. H. G. Lewin, J. van Paradijs, \& E. P. J. van den Heuvel, Cambridge Astrophys. Ser., 26, 252

Verrecchia, F., Israel, G. L., Negueruela, I., et al. 2002, A\&A, 393, 983

Wallace, L., \& Hinkle, K. 1997, ApJS, 111, 445 